\title{
Alarming Presentation of a Seemingly Common Condition in a Patient with Renal Impairment
}

\author{
Mohammad Budruddin ${ }^{1 *}$, Muhammad Yasir Khalil ${ }^{2}$, Issa Al Salmi ${ }^{2}$, Ramaiah Shilpa ${ }^{2}$ \\ ${ }^{1}$ Nephrology Department, Tawam Hospital, Al Ain, UAE \\ ${ }^{2}$ Nephrology Department, Royal Hospital, Muscat, Oman \\ Email: *drbudruddin@gmail.com
}

Received August 8, 2013; revised September 9, 2013; accepted October 2, 2013

Copyright ( 2013 Mohammad Budruddin et al. This is an open access article distributed under the Creative Commons Attribution License, which permits unrestricted use, distribution, and reproduction in any medium, provided the original work is properly cited.

\begin{abstract}
We received a 23-year-old male, working in the Army for 18 months, with advanced renal impairment, haemoptysis and hyperkalemia. An impression of the pulmonary renal syndrome was made and he was managed aggressively with haemodialysis, plasma exchange and pulsing with methyl prednisolone. His condition improved, but the renal functions did not. The vasculitic workup including Extractable Nuclear Antigen (ENA) and Anti-Neutrophil Cytoplasmic Antibody (ANCA) which were sent on admission came out to be negative. As he apparently responded to the initial management protocol, he was subjected to renal biopsy which did not reveal any vasculitis but was consistent for end-stage renal disease. Subsequently he was subjected to lung biopsy which also was not supportive for vasculitis. However, we concluded that the initial pointers for Wegener's granulomatosis were misleading. The haemoptysis entirely subsided as the congestion improved. He was maintained on Haemodialysis three times per week schedule and discharged to the peripheral health care facility.
\end{abstract}

Keywords: Haemoptysis; Renal Impairment; Pulmonary Renal Syndrome; Haemodialysis; ENA; ANCA; Vasculitis; Pulmonary Oedema

\section{Introduction}

Pulmonary oedema is not an unusual presentation in patients with end-stage renal disease. This may be associated with haemoptysis. Radiological imaging of the chest may at times be very confusing, mimicking alveolar haemorrhage. We had a young patient who presented acute onset advanced renal failure with haemoptysis. In the course of his management, we concluded that his haemoptysis was secondary to pulmonary oedema and not pulmonary renal syndrome.

\section{Case Summary}

Mr. IRS, a 23-year-old Omani male was referred from a peripheral Hospital with advanced renal failure and haemoptysis. He was not know to have any medical problems in the past and was not on any medications before this presentation. Neither was he ever told in any health care facility to be having renal impairment. He presented with the advanced renal failure with serum creatinine

${ }^{*}$ Corresponding author. more than $2000 \mathrm{mmol} / \mathrm{l}$ and urea $84 \mathrm{mmol} / \mathrm{l}$. He was in severe respiratory distress (tachypnoeic RR of 35/m, desaturating to $78 \%$ on room air) and developed massive frank haemoptysis immediately after admission. The chest X-ray Figure 1 on admission and urgent high resolution CT (HRCT) Figure 2 done after two sessions of Haemodialysis with good ultrafiltration showed extensive alveolar haemorrhage with perivascular nodules. This concurred with our initial impression of pulmonary renal syndrome (Wegener's granolomatosis). Further CT of the sinuses did show the polypoidal thickening of the mucous membrane of the right frontal sinus. The complements level done were normal.

His condition was critical with severe drop in $\mathrm{Hb}$ to 4 $\mathrm{gm} / \mathrm{dl}$. He was started on emergent aggressive treatment for Wegner's granulomatosis with 7 sessions of plasma exchange and methylprednisolone $1 \mathrm{gm}$ pulse daily for three days. His haemoptysis subsided after the forth session of plasma exchange and his general condition also improved with improvement in the pulmonary congestion and uremia following several sessions of haemodialysis and ultrafiltrations. 
The vasculitic workup subsequently came out to be negative for Extactable Neuclear antigens (ENA), AntiNeutrophil Cytoplasmic Antibody but equivocal for Anti-glomerular basement membrane antibody (Anti GBM). Serology for HBV, HCV and HIV were negative. Repeat radiology showed improvement in the alveolar shadows. Ultrasound estimation of kidney size was normal with increased ecogenicity. The urine analysis was bland (no active sediments) showed $<2$ RBC, $<2$ WBC, of 25 mg/L Protien.

After stabilizing he was subjected to renal biopsy in order to get the tissue diagnosis. The histopathology did not show any evidence of vasculitis, tubulointerstitial compartment showed marked sclerosis of the interstitium and scattered moderate inflammatory cells, tubular atrophy with dilatation. The features were consistent with the end stage renal disease (Figure 3)

Even the lung tissue biopsy done did not reveal any granuloma, pneumonic changes and evidence of vasculitis. However there were findings of scanty recent and

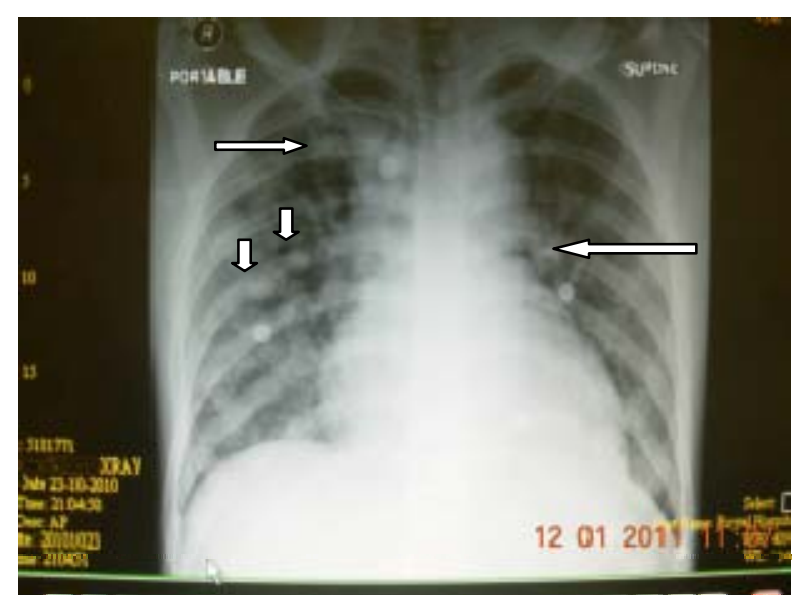

Figure 1. Chest X-ray showing nodular opacities (indicated by small and big arrows).

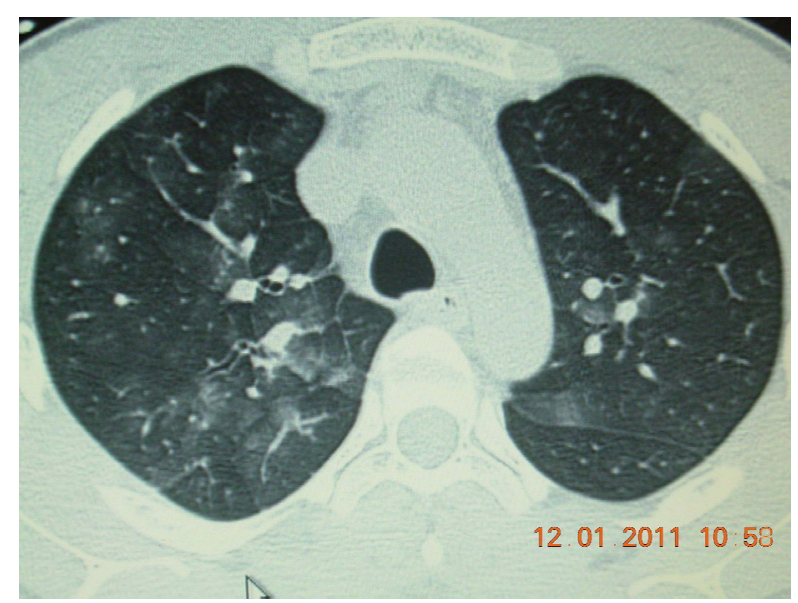

Figure 2. CT scan of the chest showing ground glass appearance. old intra-alveolar haemorrhage (5\% of the tissue sample) (Figure 4)

Semi-permanent vascular access (Right Internal Jugular vein tunneled catheter) was inserted for him and he was put on maintenance haemodialysis three times per week and radiocephalic fistula was also created for him. He was discharged to the peripheral health care facility with dialysis facility, in a stable condition with a plan for living related renal transplantation from his brother in the future.

\section{Discussion}

The presentation with pulmonary oedema is very common in patients on maintenance haemodialysis. These end stage renal disease patients irrespective of etiology are at times polyuric before being started on dialysis. However after initiating on haemodialysis they tend to decrease their urine output. Little excess of fluid intake inter dialytic period leads to pulmonary congestion and at times frank pulmonary oedema. This is exacerbated by the concurrent presence of poorly functioning heart.

In our patient the scenario was a bit perplexing as he presented for the first time with very advanced renal failure and pulmonary congestion. However his urine

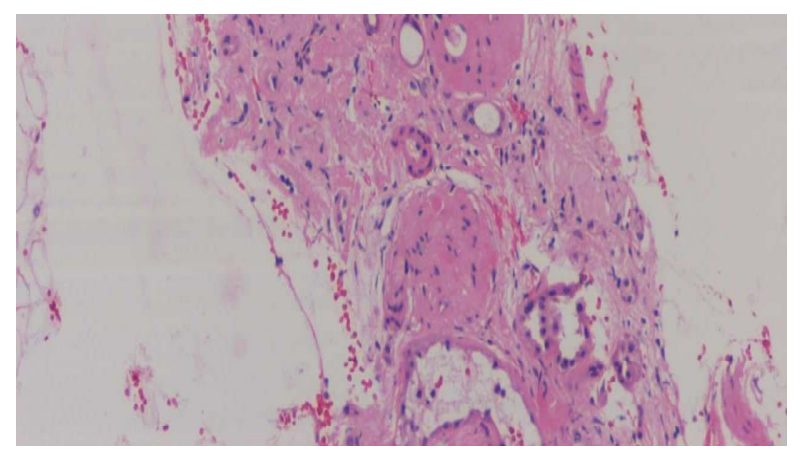

Figure 3. Haematoxylin Eosin stain section shows a core of kidney tissue with two globally hyalinised glomeruli, (black arrow heads) marked tubulointerstitial sclerosis and mild chronic inflammation.

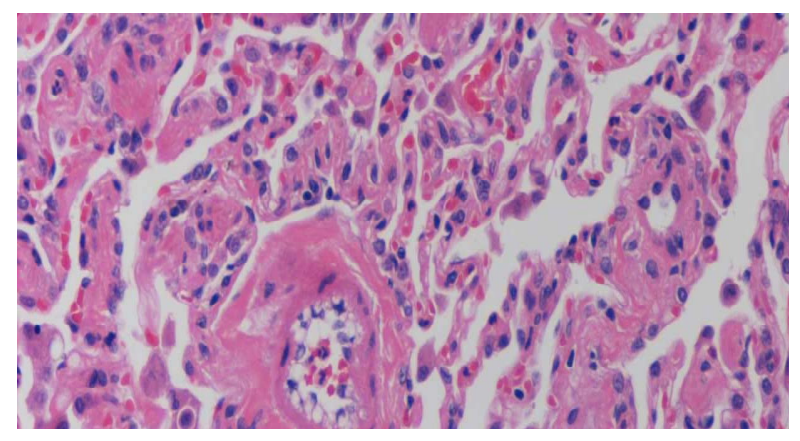

Figure 4. Section showing the lung tissue with mild thickening of the alveolar septa and congestion. No vasculitis or granuloma seen. 
output was nearly $1500 \mathrm{ml}$ in 24 Hours. The clinical presence of frank haemoptysis with first time documentation of grossly deranged renal functions and CT finding of alveolar haemorrhage was quite suggestive of pulmonary renal syndrome (Wegener's Granulomatosis/Good pastures syndrome).

Pulmonary oedema can at times present with haemoptysis but again it usually does not show alveolar haemorrhages.

However going through the literature we realized that radiological findings of pulmonary oedema can present in several forms [1].

a) Postobstructive pulmonary edema. lism.

b) Pulmonary edema with chronic pulmonary embo-

c) Pulmonary edema with veno-occlusive.

d) Stage 1 near drowning pulmonary edema manifests as Kerley lines, peribronchial cuffing, and patchy, perihilar alveolar areas of airspace consolidation;

e) stage 2 and 3 lesions are radiologically nonspecific.

f) Pulmonary edema following administration of cytokines.

g) High-altitude pulmonary edema.

(h) Neurogenic pulmonary edema manifests as bilateral, rather homogeneous airspace consolidations that predominate at the apices in about $50 \%$ of cases.

i) Reperfusion pulmonary edema.

j) Postreduction pulmonary edema manifests as mild airspace consolidation involving the ipsilateral lung.

k) Pulmonary edema due to air embolism initially demonstrates interstitial edema followed by bilateral, peripheral alveolar areas of increased opacity that predominate at the lung bases.

The typical alveolar shadows seen in our patients CT is one of the forms.

Further it is well known that those patients with obstructive airway disease who develop congestive heart failure have much higher pulmonary extravascular volume. Left ventricular failure due to hypoxia leads to left atrial hypertension, which could be the patho-physiologic basis of interstitial pulmonary oedema [2].

Histological studies in uremic lungs have proven the presence of proteinaceous oedema fluid though many have even shown intra-alveolar haemorrhage [3]. Evaluation of the oedema fluid by direct endotracheal aspiration have even shown high content of protein which could suggest increased pulmonary vascular permeability to plasma proteins in renal failure [4]. Though a common feature of pulmonary oedema is increased permeability of the basement membrane to water and low molecular weight solutes, this may be attributable to the hemodynamic factors playing role in the alveolar interstitium and basement membrane [5].

\section{Conclusion}

Patients with pulmonary edema may present not only blood tinged frothy sputum but may even present massive haemoptysis, which may be life threatening and mimicking serious medical conditions, clinically and radiologically. Keeping this in mind one must deal cautiously with patients presenting pulmonary haemorrhage (haemoptysis) and advanced renal failure.

\section{REFERENCES}

[1] T. Gluecker, P. Capasso, P. Schnyder, F. Gudinchet, M.-D. Schaller, J.-P. Revelly and R. Chiolero, "Clinical and Radiologic Features of Pulmonary Edema,” Radio Graphics, Vol. 19, No. 6, 1999, pp. 1507-1531. http://dx.doi.org/10.1148/radiographics.19.6.g99no21150 $\underline{7}$

[2] R. M. McCredie, "Pulmonary Oedema in Lung Disease," British Heart Journal, Vol. 32, No. 1, 1970, pp. 66-70. http://dx.doi.org/10.1136/hrt.32.1.66

[3] L. Doniach, "Uremic Oedema of the Lungs," The American Journal of Roentgenology, Radium Therapy and Nuclear Medicine, Vol. 58, No. 5, 1947, pp. 620-628.

[4] E. C. Rackow, I. A. Fein, C. Sprung, R. S. Grodman, "Uremic Pulmonary Oedema," American Journal of Medicine, Vol. 64, No. 6, 1978, pp. 1084-1088.

[5] D. G. Gibson, "Hemodynamic Factors in the Development of Acute Pulmonary Oedema in Renal Failure," The Lancet, Vol. 288, No. 7475, 1966, pp. 1217-1220. http://dx.doi.org/10.1016/S0140-6736(66)92302-6 